\title{
Magnetic resonance imaging findings in pulmonary Kaposi's sarco- ma: a series of 10 cases
}

\author{
A.M. Khalil*, M.F. Carette*, J.L. Cadranel**, Ch.M. Mayaud**, \\ G.M. Akoun**, J.M. Bigot*.
}

Magnetic resonance imaging findings in pulmonary Kaposi's sarcoma: a series of 10 cases. A.M. Khalil, M.F. Carette, J.L. Cadranel, Ch.M. Mayaud, G.M. Akoun, J.M. Bigot. CERS Journals Ltd 1994.

ABSTRACT: Since chest X-ray and CT scan features of Kaposi's sarcoma (KS) are nonspecific, we wanted to test the hypothesis that the histological components of this tumour and/or the associated haemorrhagic component, may result in a characteristic signal pattern on magnetic resonance imaging (MRI).

Thoracic MRI was performed in a prospective manner in ten patients with acquired immune deficiency syndome (AIDS) and pulmonary KS. MRI examinations (1.5 Tesla) included Spin-echo T1 (SE-T1), before and after gadolinium injection, as well as T2-weighted sequences (SE-T2). For each sequence the signal intensity of lesions was measured and compared with each other as well as with the signal intensity of muscle. Results were compared to the hemosiderin content of macrophages in the bronchoalveolar lavage (BAL) in all patients and with histological findings in three. The results were compared to values obtained in a control group of seven patients with pneumocystis carinii pneumonia.

SE-T1 showed focally increased signal intensity in the pulmonary parenchyma $(n=5)$. Signal enhancement in parenchymal lesions $(n=10)$ and along peribronchovascular trees $(n=5)$ was observed after gadolinium injection. The second echo of SE-T2 showed a markedly reduced signal intensity in pathologic areas $(n=10)$. This last finding was not observed in the control group.

In conclusion, we have identified a pattern of MRI signal abnormalities suggestive of Kaposi's sarcoma. The MRI signal intensity of KS lesions may be related to the angiomatous and fibrous components of the tumour. A correlation between hemosiderin deposits and signal intensity was not demonstrated in this study, perhaps because of a poor correlation between BAL results and the actual occurrence of an haemorragic component in parenchymal lesions.

Eur Respir J., 1994, 7, 1285-1289.
*Dept of Radiology. **Dept of Clinical and Intensive Care Respiratory Diseases, Tenon hospital, 4 rue de la chine 75020 Paris, France.

\author{
Correspondence: M.F. Carette \\ Department of Radiology \\ Tenon hospital \\ 4 rue de la chine \\ 75020 Paris \\ France
}

Keywords: Acquired immunodeficiency syndrome

Kapsosi's sarcoma

lung

magnetic resonance imaging

Received: April 261993

Accepted after revision March 251994
Since 1981, Kaposi's sarcoma (KS) has gained considerable interest due to its endemic occurrence in patients with the acquired immune deficiency syndrome (AIDS). In the AIDS population, $\mathrm{KS}$ is a multicentric malignant process that frequently affects lymph nodes and the gastrointestinal tract. Lesions also occur in the lung and are a cause of pulmonary infiltrates and respiratory failure. Accurate diagnosis is essential because chemotherapy appears to provide some palliation. The histological features of this tumour include an angiomatous component and a fibrous component (spindle cells forming rudimentary fascicles). Moreover, a clinicopathological study of AIDS patients with KS showed that alveolar haemorrhage occurred more frequently in these patients than in other AIDS patients with different lung pathologies [1].

Chest X-ray features of KS have been well described [2-8]. The most common pattern is bilateral reticulonodular infiltrates, mainly in the lower lobes, associated with pleural effusions. CT scan provides more specific information than chest X-ray for identification of pulmonary KS [9-11]. CT also allows better delineation of the abnormal hilar densities which extend into the adjacent pulmonary parenchyma, the peribronchovascular wall thickening, the poorly delineated parenchymal masses, and the frequent bilateral pleural effusions. However, these findings are again not specific.

In an attempt to find out if the histological characteristics of this tumour could result in a particular pattern of signal abnormalities in MRI, we performed a prospective study in 10 patients with pulmonary KS. Seven patients with pneumocystis carinii pneumonia (PCP) were used as controls.

\section{Patients and methods}

We performed a prospective thoracic MRI study in ten AIDS patients with pulmonary KS from July 1991 to February 1992. Patients' ages ranged from 21-44 yrs (mean age $33 \mathrm{yrs}$ ). They were all male: nine homosexual 
and one bisexual. Symptoms consisted of cough and dyspnoea in nine patients and haemoptysis in one patient. No patient had had PCP within a six month period prior to MRI examination. Two patients had cytomegalovirus (CMV) in BAL fluid without any other sign of CMV infection. In three patients the diagnosis of $\mathrm{KS}$ was established histologically by transbronchial biopsy, open lung biopsy, and mediastinoscopy with lymph node biopsy, respectively; microscopic findings included an angiomatous component and a fibroblastic component. In two of these three an important haemorrhagic component was observed. One was an alveolar haemorrhage (case 1), the other was located in mediastinal lymph nodes (case 3 ). The remaining seven cases had biopsy proven skin lesions, characteristic endobronchial lesions $(n=7)$, compatible findings on chest X-ray or CT (fig 1a) and no evidence of infection [9-13].

Fibreoptic bronchoscopy, bronchoalveolar lavage (BAL), and bronchial and transbronchial biopsies were performed in all patients. Bronchial and transbronchial biopsies provided a histological diagnosis in only one patient. BAL fluid was examined by cytological and microbiological methods with respect to bacteria (including mycobacteria), parasites, fungi and viruses.

a)

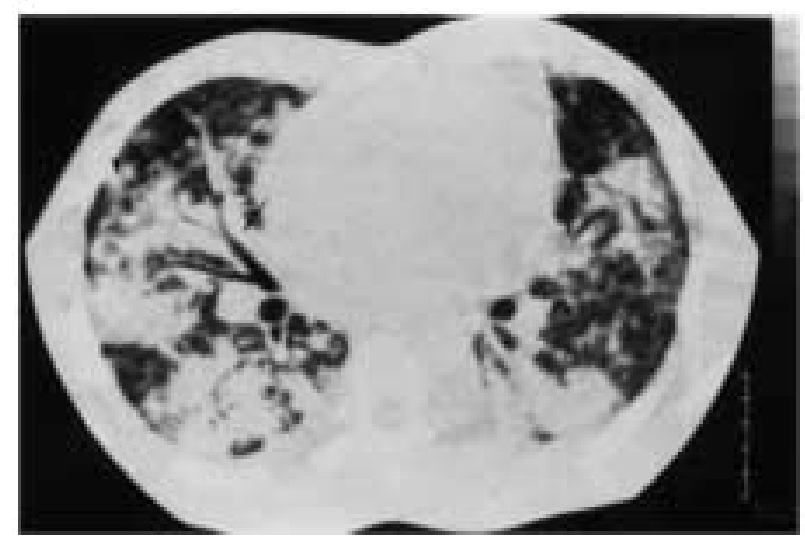

c)

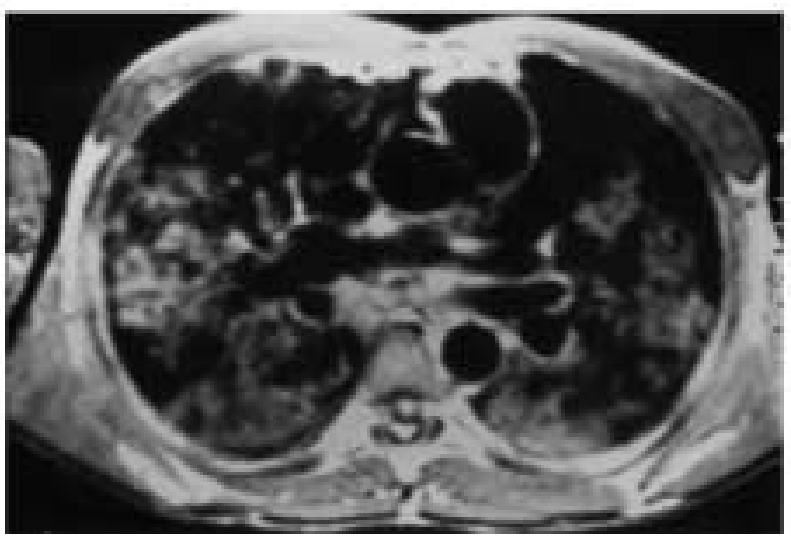

Alveolar haemorrhage was demonstrated in nine patients.

The hemosiderin content of alveolar macrophages was estimated on Perls' stained slides (iron staining) on a three point scale as follows: $(+)$ with a little blue staining; $(++)$ with mild colour intensity and $(+++)$ with marked blue staining.

The control group consisted of seven AIDS patients with PCP, all male, 4 of whom were homosexual. The age range was 24-53 yrs (mean: 35 yrs). The diagnosis of PCP was based on BAL in four cases, on BAL and open lung biopsy (OLB) results in one case, on BAL and autopsy in one case. In one case, only the post mortem examination was positive. Radiological features included diffuse interstitial involvement with ground glass opacities in five patients; one patient had predominantly apical changes and a cavitating lesion leading to an OLB. In the two other cases, atypical findings with cystic lesions or pleural and pericardial effusions attributed to PC were demonstrated on post mortem examination only. The MRI examinations were performed prior to our KS studies. The same sequences were obtained but without contrast injection as gadolinium was not yet available at that time. Data for retrospective signal evaluation were available in six

b)

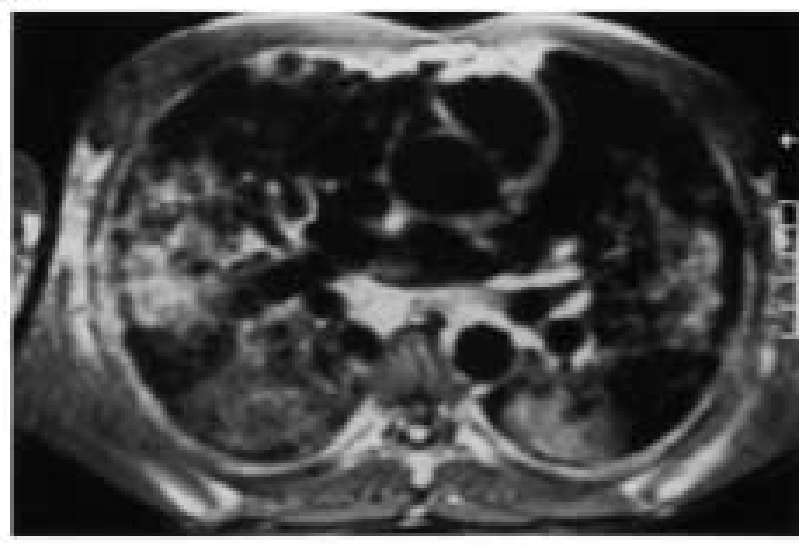

d)

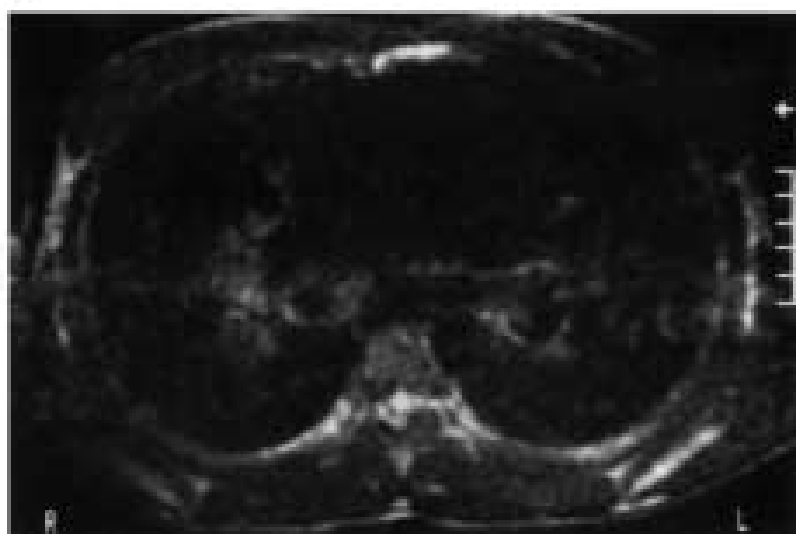

Fig. 1. - Transverse thoracic scan in a patient with pulmonary Kaposi's sarcoma: a) CT scan, slice of $2 \mathrm{~mm}$ through the middle lobe. Thickened perivascular and peribronchial walls (arrow) with bilateral disseminated nodules and tumour (head arrow). b) T1-weighted magnetic resonance (MR) image $(T E=20 \mathrm{~ms} / T R=650 \mathrm{~ms})$. Diffuse increased signal intensity in the lower part of right and left lung. c) T1-weighted MR image after gadolinium i.v. injection. Enhancement of signal intensity in the lesions. d) T2-weighted MR image (TE $=100 \mathrm{~ms} / \mathrm{TR}=1800 \mathrm{~ms}$ ). Markedly reduced signal intensity in the same area. CT: computed tomography. 
patients. All measurements were performed with the same method as for patients with KS.

MRI were obtained with a 1.5 Tesla unit (Gyroscan S15 HPP Philips) with a body coil. All examinations included four multislice spin-echo (SE) sequences. The first sequence was cardiac gated T1-weighted with TE $=20 \mathrm{~ms}$ and four excitations in the frontal plane. The next three sequences were performed in the transversal plane, all with the same centre, chosen from the findings of the first sequence. It was always in the lower part of the thorax. The three sequences were: a cardiac gated T1-weighted sequence with $\mathrm{TE}=20 \mathrm{~ms}$ and four excitations, a T2-weighted sequence with $\mathrm{TE}=50 / 100 \mathrm{~ms}$ and $\mathrm{TR}=1800 \mathrm{~ms}$ and two excitations, and the same as the first transversal sequence but after i.v. injection of contrast medium (gadopentetate-dimeglumine $0.2 \mathrm{ml} \cdot \mathrm{kg}^{-1}$ ).

Slices were $10 \mathrm{~mm}$ thick with a gap of $1 \mathrm{~mm}$; matrix size was $256 \times 256$ and the field of view was $450 \mathrm{~mm}$. No ventilation or flow compensation was used.

Our quantitative data were obtained solely from the transversal slices. The frontal plane was used only for anatomic delineation and for choosing the level of the three transversal slices. Signal intensities of lesions, muscles, and fat tissue were measured in the same area for each sequence. The area of the region of interest (ROI) was about $1 \mathrm{~cm}^{2}$. Signal intensity ratios were calculated comparing signal of lesions to signal of muscles, for the same sequences. We also compared signal intensities of lesions and ratios between sequences. The relationship between these data and the visual impression was performed. We also compared MRI findings with BAL results as for hemosiderin content.

\section{Results}

On T1-weighted sequences, high signal intensity in the parenchymal lesions was visually observed in five cases (fig. 1b). This high signal intensity was reflected by the high signal intensity ratio of lesion to muscle in four cases (fig. 2) and only by image analysis and visual estimation in one case because of the irregular and patchy distribution of the areas of high signal intensity. Signal abnormalities were caused by small

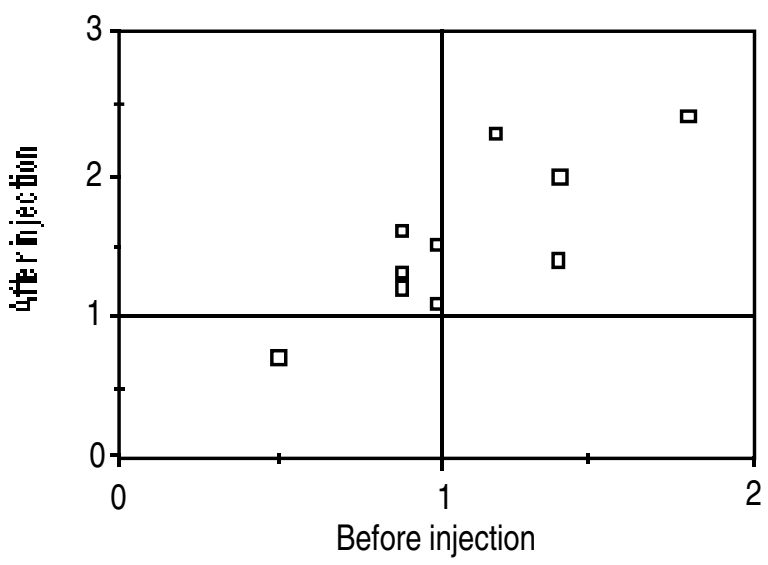

Fig. 2. - Signal intensity ratio of lesion to muscle before and after gadolinium injection in ten patients with Kaposi's sarcoma.

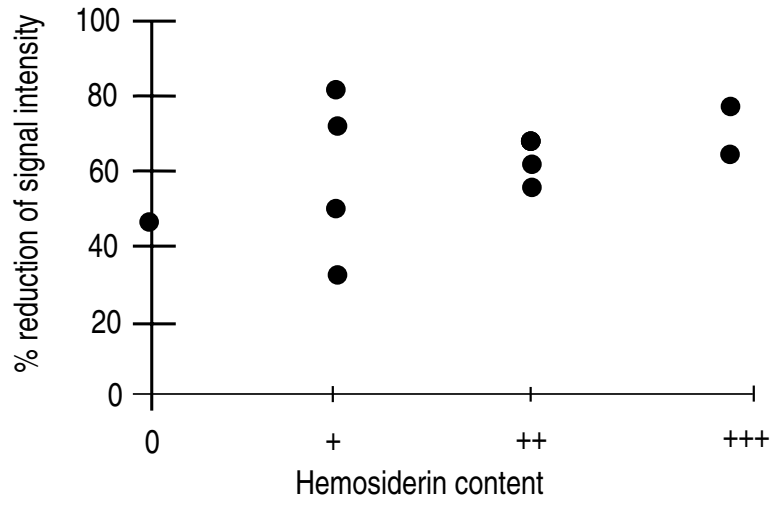

Fig. 3. - Hemosiderin content of macrophages found in BAL from 10 patients with KS compared to percentage of reduction of signal intensity between T1-weighted and T2E2-weighted sequence. 0: no colouration; +: a little blue staining; ++: mild colour intensity; +++: marked blue staining.

areas of high signal intensity in lesions having a signal isointense to that of muscle. Signal intensity of parenchymal lesions in the five remaining patients was almost similar to the muscle signal. Mean of standard deviation for signal evaluation in T1-weighted sequences was $8.7 \%$ of signal value.

On T1-weighted sequences after gadolinium injection, marked enhancement in signal intensity of parenchymal lesions was observed in eight patients (fig. 1c); moderate enhancement was observed in the other two patients. Contrast enhancement was also observed along perivascular and peribronchial pathways in 5 patients with a concomitant enhancement of the oesophageal wall signal in four. This enhancement is well displayed when we compared signal intensity ratios of KS lesions to muscle (fig 2).

On the first echo (E1) of T2-weighted images (TE=50ms) we observed an increased signal intensity of lesions in comparison with T1-weighted images in only three patients. In the other seven patients signal intensity of lesions was equal to or slightly below that of T1-weighted images. On the second echo (E2) of T2-weighted images $(\mathrm{TE}=100 \mathrm{~ms})$ we observed a markedly reduced signal intensity in the areas of pathological lesions in all cases (fig. 1d). There was a 30 to $50 \%$ signal intensity reduction in three patients, 50 to $60 \%$ reduction in three cases and up to $70 \%$ reduction in four patients (fig. 3). Comparison between these percentages of signal reduction and estimation of hemosiderin content of macrophages in each case had no significant correlation (fig. 3).

In the seven control patients, areas of high signal intensity were also observed in the parenchyma $(n=6)$ with areas of medium signal on T1-weighted sequence. In two patients where cavities were observed, the internal wall of these cavities had a very bright signal (fig. 4a). Images from T2-weighted sequence were available in 6 patients. Considering only diffuse parenchymal lesions, excluding the very bright signal of cavity walls, of lymph nodes or of pleural and pericardial effusions, a high signal intensity was observed in three cases (figs. $4 \mathrm{~b}$ and $5 \mathrm{a}, \mathrm{b}$ ). 
a)

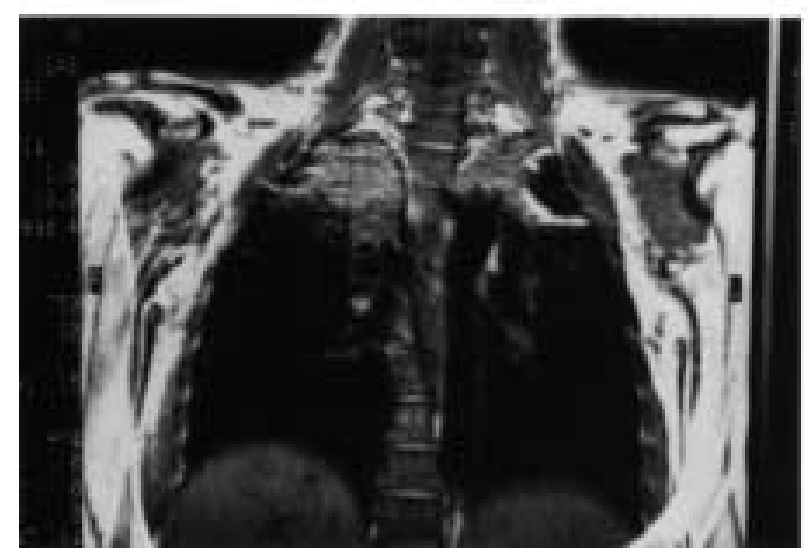

b)

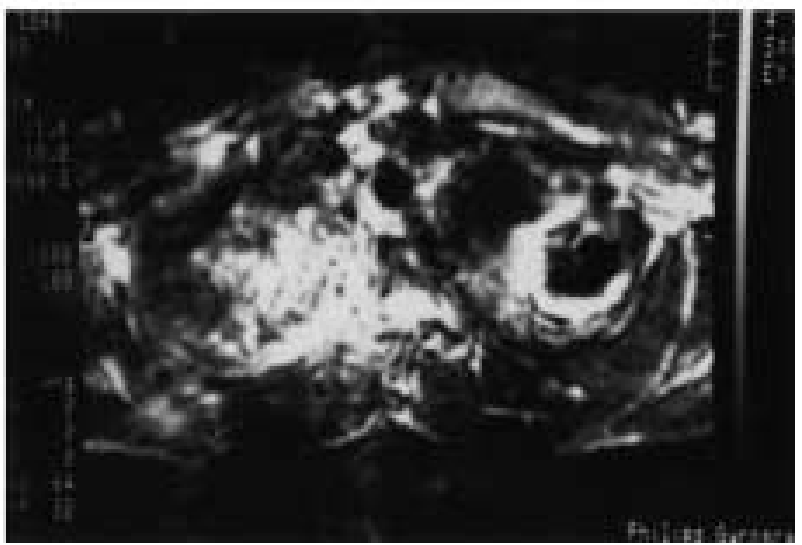

Fig. 4. - MR images in a $24 \mathrm{yr}$ old male having an atypical form of pneumocystis carinii pneumonia (PCP) including bilateral apical opacities and a cavitating lesion in the left upper lobe. This PCP was proven on open lung biopsy. a) T1-weighted frontal image (TE=30ms/TR=750ms). Diffusely increased signal intensity in the upper lobes. Very bright signal from the internal wall of the cavity. b) T2-weighted transversal image ( $\mathrm{TE}=100 \mathrm{~ms} / \mathrm{TR}=1800 \mathrm{~ms})$. Markedly increased signal intensity in the upper lobes.

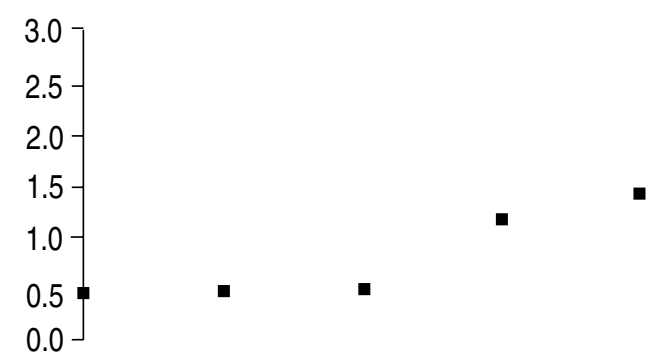

b)

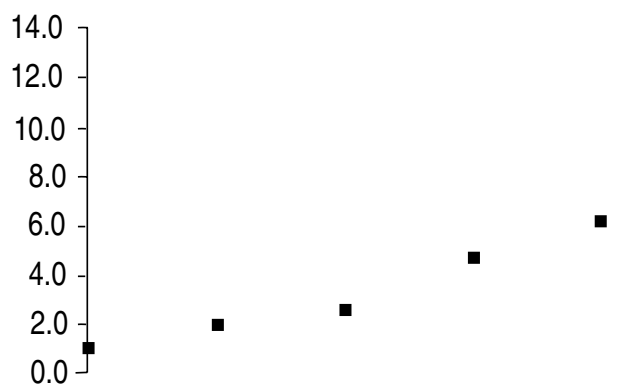

Fig. 5. - Control group of seven AIDS patients with Pneumocystic carinii pneumonia (PCP). Signal intensity ratio of a) lesion in T2E2 to lesion in T1 sequence; b) lesion to muscle in T2E2 sequence.

\section{Discussion}

Kaposi's sarcoma occurs in about $50 \%$ of patients with AIDS in autopsy series [14-15]. Involvement of pulmonary parenchyma and airways is common and generally follows involvement of skin, gastrointestinal tract, and liver. Disease of the visceral pleura has also been described, with or without parenchymal involvement. In clinical studies, pulmonary KS involvement is estimated to account for 6 to $12 \%$ of all AIDS pulmonary comp- lications $[2,4,5]$. At autopsy, frequency rises to $30 \%$ [14-17].

Because of technical limitations, MRI has not been used extensively in the investigation of thoracic disease. Artifacts generated by cardiac and respiratory movements are difficult to control and the parenchyma is difficult to visualize because of the numerous air-tissue interfaces inherent in the structure of the lung. The technology is, as yet, not capable of overcoming the inherent magnetic susceptibility of the lung [18].

Only a few studies have reported thoracic MRI findings in AIDS patients [19] and there have been no previous studies on pulmonary KS.

In our study on T1-weighted sequences, parenchymal lesions, thickening along the peribronchovascular sheaths, and pleural effusions were well demonstrated. Parenchymal lesions appear as areas of medium signal or of mixed medium and high signal intensity as in control patients. Cavities were not observed in KS. Thickening of peribronchial and perivascular structures was observed in $50 \%$ of cases. These abnormalities generated a signal of medium or high intensity similar to that from parenchymal lesions.

Hence, T1-weighted images before injection of contrast medium did not demonstrate significant differences between KS and PCP. The presence of anatomical differences, peribronchovascular thickening in $\mathrm{KS}$ and cysts in PCP, was better displayed on CT.

The increase of signal intensity, after gadolinium injection, particularly in lesions with peribronchovascular distribution was highly suggestive of the diagnosis of KS. The enhancement may be related to the angiomatous component of the tumour which contains numerous dilated, irregularly shaped capillary vessels [11-20].

Our study demonstrated that KS lesions have a low signal on T2-weighted sequences in contrast to the lesions in the patients of the control group. The reduced signal intensity in KS could depend on either alveolar haemorrhage or the fibrous component of the tumour. 
Which of these explanations is dominating cannot be established from this small series, in which biopsy of the lesions was obtained in only 3 cases. A decrease of the signal from pulmonary lesions on T2-weighted sequences have been reported, however, in one case of idiopathic pulmonary hemosiderosis [21] and in one case of pulmonary haemorrhage complicating systemic lupus erythematosus [22]. However, we found no significant correlation between the hemosiderin content of macrophages and percentage of signal reduction between T1 and E2T2 (fig. 3). This may be due to poor correlation between hemosiderin content of the macrophages in BAL and the actual haemorrhagic component of the lesions, as in one case in whom a hemorrhagic component was demonstrated by open lung biopsy although the BAL was negative for hemosiderin. Similar findings were observed in three patients in the clinicopathological study by FOURET et al. [1]. Comparison with the control group is very interesting because an increase in signal intensity was observed on T2E2 images in three cases (figs. $4 \mathrm{~b}$ and $5 \mathrm{a}, \mathrm{b}$ ); these differing results in the control group eliminate the possibility of the technical problem of low signal to noise ratio causing artefactual results in our KS population.

In conclusion, the MRI features of pulmonary Kaposi's sarcoma are: irregular increased signal intensity in T1-weighted images; markedly reduced signal intensity on T2-weighted images, and strong lesional contrast enhancement after administration of gadolinium. This pattern of signal abnormalities, particularly when seen in a peribronchovascular distribution, is suggestive of Kaposi's sarcoma. MRI may provide a specific and noninvasive method for diagnosis of KS. Further studies, including various aetiologies of pulmonary disease in AIDS patients, are needed to test the specificity of our findings.

\section{References}

1. Fouret PJ, Touboul JL, Mayaud CM, Akoun GM, Roland J. Pulmonary Kaposi's sarcoma in patients with acquired immune deficiency syndrome: a clinicopathological study. Thorax 1987; 42: 262-268.

2. Davis SD, Henschke CI, Chamides BK, Westcott JL. Intrathoracic Kaposi sarcoma in AIDS patients: Radiographic - pathologic correlation. Radiology 1987; 163: 495-500.

3. Garay SM, Belenko M, Fazzini E, Schinella R. Pulmonary manifestations of Kaposi's sarcoma. Chest 1987; 91: 39-43.

4. Hill CA, Harle TS, Mansell PWA. The prodrome, Kaposi sarcoma, and infections associated with acquired immunodeficiency syndrome: Radiologic findings in 39 patients. Radiology 1983; 149: 393-399.

5. McCauley DI, Naidich DP, Leitman BS, Reede DL, Laubenstein L. Radiographic patterns of opportunistic
Lung infections and Kaposi sarcoma in homosexual men. Am J Roengenol 1982; 139: 653-658.

6. Meduri GU, Stover DE, Lee M, Myskowski PL, Caravelli JF, Zaman MB. Pulmonary Kaposi's sarcoma in the acquired immune deficiency syndrome. Clinical, radiographic, and pathologic manifestations. Am J Med 1986; 81: 11-18.

7. Ognibene FP, Steis RG, Macher AM, et al. Kaposi's sarcoma causing pulmonary infiltrates and respiratory failure in the acquired immunodeficiency syndrome. Ann Intern Med 1985; 102: 471-475.

8. Zibrak JD, Silvestri RC, Costello P, et al. Bronchoscopic and radiologic features of Kaposi's sarcoma involving the respiratory system. Chest 1986; 90: 476-479.

9. Naidich DP, Tarras M, Garay SM, Birnbaum B, Rybak BJ, Schinella R. Kaposi's sarcoma: CT-Radiographic correlation. Chest 1989; 96: 723-728.

10. Carette MF, Mayaud Ch, Bigot JM. Pulmonary and mediastinal involvement. In: P.M. Trotot, B.C. Decker eds. "Imaging of AIDS". Philadelphia 1991: 25-56.

11. Wolff SD, Kuhlman JE, Fishman EK. Thoracic Kaposi sarcoma in AIDS: CT findings. $J$ Comput Assist Tomogr 1993: 17; 60-62.

12. White DA, Matthay RA. Noninfectious pulmonary complications of infection with the human immune deficiency virus. Am Rev Respir Dis 1989; 140: 1763-1787.

13. Murray JF, Garay SM, Hopewell PC, Mills J, Snider GL, Stover DE. Pulmonary complications of the acquired immunodeficiency syndrome: An Update. Am Rev Respir Dis 1987; 135: 504-509.

14. Welch K, Finkbeiner W, Alpers CE, et al. Autopsy findings in the acquired immune deficiency syndrome. JAMA 1984; 252: 1152-1159.

15. Lamoureux E, Urmacher C. Pulmonary involvement in AIDS. Results of post-mortem examination in fifty cases. (in French). Sem Hôp Paris 1986; 62: 3301-3305.

16. Nash G, Fligiel S. Pathologic features of the lung in the acquired immune deficiency syndrome (AIDS): An autopsy study of seventeen homosexual males. Am J Clin Pathol 1984; 81: 6-12.

17. Reichert CM, O'Leary TJ, Levens DL, Simrell CR, Macher AM. Autopsy pathology in the Acquired Immune deficiency Syndrome. Am J Pathol 1983; 112: 357-382.

18. Bergin CJ, Nol DC, Pauly JM, Glover GH, Macovski A. MR imaging of lung parenchyma: a solution to susceptibility. Radiology 1992; 183: 673-676.

19. Mac Fadden RG, Carr TJ, Mackie IDF. Thoracic magnetic resonance imaging in the evaluation of HIV-l/ AIDS pneumonitis. Chest 1992; 101: 371-374.

20. Sivit CJ, Schwartz AM, Rockoff SD. Kaposi's sarcoma of the lung in AIDS: Radiologic-Pathologic analysis. Am J Roengenol 1987; 148: 25-28.

21. Rubin GD, Edwards DK, Reicher MA, Doemeny JM, Carson SH. Diagnosis of pulmonary hemosiderosis by MR Imaging. Am J Roengenol 1989; 152: 573-574.

22. Hsu BY, Edwards DK, Trambert MA. Pulmonary hemorrhage complicating systemic lupus erythematous: role of MRI in diagnosis. Am J Roengenol 1992; 158: 519-520. 